Cite this: J. Mater. Chem. A, 2013, 1, 12345

Received 17th June 2013

Accepted 13th August 2013

DOI: $10.1039 / \mathrm{c} 3 \operatorname{ta} 12358 \mathrm{~h}$

www.rsc.org/MaterialsA

\section{Charge carrier transport and contact selectivity limit the operation of PTB7-based organic solar cells of varying active layer thickness $\uparrow$}

\author{
Antonio Guerrero, ${ }^{a}$ Nuria F. Montcada, ${ }^{\mathrm{b}}$ Jon Ajuria, ${ }^{\text {cd }}$ Ikerne Etxebarria, ${ }^{\text {cd }}$ \\ Roberto Pacios, ${ }^{c d}$ Germà Garcia-Belmonte ${ }^{* a}$ and Emilio Palomares ${ }^{* b e}$
}

In this work we study the different electrical loss pathways occurring during the operation of bulk heterojunction solar cells by using a variety of electrical and optical characterization techniques beyond the current density-voltage curve $(J-V)$ : Impedance Spectroscopy (IS), Charge Extraction (CE) and Transient Photovoltage (TPV). Two sets of devices are analyzed: the first is based on the donor polymer $\mathrm{P} 3 \mathrm{HT}$, known to provide efficient cells using thick active layers (i.e. $270 \mathrm{~nm}$ ), and the recently developed PTB7 which offers maximum efficiencies for devices with thinner layers (i.e. $100 \mathrm{~nm}$ ). Devices fabricated with $\mathrm{P} 3 \mathrm{HT}: \mathrm{PC}_{60} \mathrm{BM}$ are not limited by transport of carriers and large active layer thickness may be used. Importantly, increasing the active layer thickness does not modify the contact selectivity. This is supported by analysis of the diode curve measured in the dark (similar leakage currents) and by capacitance-voltage measurements (similar fullerene content covering the cathode). Under these conditions the current density curve under illumination is mainly defined by the recombination processes taking place in the bulk of the active layer. In contrast, transport of carriers and contact selectivity are both limiting factors for the PTB7:PC ${ }_{60} \mathrm{BM}$ system. In this case, best efficiencies are obtained with a low active layer thickness and a high fullerene ratio. Reduced active layer thickness minimizes undesired electrical resistances related to carrier transport through the bulk of the active layer. High fullerene content enhances the amount of fullerene molecules at the cathode leading to decreased leakage currents. Then, the overall device efficiency will be a combination of the recombination kinetics in the bulk of the active layer, undesired resistance to transport of carriers and leakage current present due to low selectivity of the contact. The use of additives has also been explored which enhances charge generation and extraction. Overall, this work provides a comprehensive guide on how to interpret results obtained from some of the most widely used optoelectronic techniques employed to analyse operating devices.

\section{Introduction}

Bulk-heterojunction (BHJ) organic photovoltaic (OPV) devices have attracted considerable attention over the last few years due to their potential to obtain high light-to-energy conversion efficiencies. The research in OPVs has been mainly focused on three fronts: first, the design and synthesis of new semiconducting polymers; ${ }^{1-3}$ second, the understanding on how the

${ }^{a}$ Photovoltaic and Optoelectronic Devices Group, Departament de Física, Universitat Jaume I, ES-12071 Castelló, Spain. E-mail: garciag@fca.uji.es; Tel: +34964387548

${ }^{b}$ Institute of Chemical Research of Catalonia (ICIQ), Avda. Països Catalans 16, Tarragona,ES-43007, Spain.E-mail: epalomares@iciq.es; Tel: +34977920241

'IK4-IKERLAN, Goiru Kalea, 20500 Arrasate, Spain

${ }^{d}$ CIC microGUNE, 20500 Arrasate, Spain

${ }^{e}$ ICREA. Passeig Lluís Companys, 23. Barcelona ES-08010, Spain

$\dagger$ Electronic supplementary information (ESI) available. See DOI: $10.1039 / \mathrm{c} 3 \operatorname{ta} 12358 \mathrm{~h}$ charge transfer processes take place and how these interfacial processes limit the device efficiency; ${ }^{\mathbf{4}, 5}$ and last but not least, closing the gap between efficiencies obtained in the laboratory and industrial processing. ${ }^{6}$

Regarding materials, the most studied and best understood system is based on the polymer poly(3-hexylthiophene) (P3HT) and fullerene $[6,6]$-phenyl- $\mathrm{C}_{61}$-butyric acid methyl ester $\left(\mathrm{PC}_{60} \mathrm{BM}\right)$. The former is used as an electron donor material and as an effective hole transport medium, and the fullerene is used as an electron acceptor and electron transport moiety. Optimization of this system yields efficiencies in the range of 3.5-4\% obtained by many research groups. A breakthrough arising from the materials development has recently been obtained with the use of the donor material poly[[4,8-bis[(2-ethylhexyl)oxy]benzo[1,2- $\left.b: 4,5-b^{\prime}\right]$ dithiophene-2,6-diyl][3-fluoro-2-[(2-ethylhexyl)carbonyl]thieno[3,4- $b]$ thiophenediyl]] (PTB7). This semiconducting polymer has led to world record efficiencies of 9.2\%. ${ }^{7}$ PTB7 combines low bandgap light absorption which 
renders the polymer capable of extending the capture of photons towards the near-IR region, and adequate energy level positions with respect to the most common selective contacts and reasonably high carrier mobility for low active layer thickness. $^{8}$

There are several techniques that can provide information on electrical parameters in operating devices. These techniques include photo-induced charge transfer measurements such as charge extraction (CE) and/or transient photovoltage (TPV).,9-11 The former provides valuable information on the charge distribution at different applied biases and the number of charges that the system is capable of extracting and accumulating. Additionally, TPV experiments can be carried out with the aim of studying the carrier recombination dynamics and carrier lifetime dependency on voltage. Alternatively, a purely electrical technique such as impedance spectroscopy (IS) has become very useful to study all resistive processes taking place in an operating device such as recombination or transport mechanisms., ${ }^{5,12,13}$ All three techniques have previously helped to better understand the origin of the open circuit voltage, ${ }^{\mathbf{1 4 - 1 8}}$ or the relationship between the bulk-heterojunction morphology and the solar cell efficiency. ${ }^{19,20}$

In this paper, we analyze the response of $\mathrm{BHJ}$ comprising either P3HT or PTB7 as an electron donor material and fullerene derivatives such as $\mathrm{PC}_{60} \mathrm{BM}$ and $\mathrm{PC}_{70} \mathrm{BM}$ as an electron acceptor moiety using all techniques aforementioned. The $\mathrm{BHJ}$ device configuration differs in the electron donor:acceptor ratio and photo-active film thickness as listed in Table 1. As will be shown later, devices limited by transport of carriers exhibit an additional electrical resistance that limits charge extraction processes. In these devices additional calculations to estimate the total series resistance become of utmost importance to comprehend correctly the device electrical characteristics. Additionally, capacitance-voltage measurements evidence different fullerene concentrations at the cathode for the different processing conditions that certainly influence the contact selectivity. Indeed, all results obtained from the optoelectronic techniques used in this work (CE, TPV or IS) reveal that the contact selectivity is a determining mechanism of the operation of the photovoltaic devices.

In particular, we observe that the two systems under study behave differently not only in terms of transport of carriers but also in terms of contact selectivity. Devices fabricated with P3HT: $\mathrm{PC}_{60} \mathrm{BM}$ are not limited by carrier transport. Moreover an increase in the active layer thickness does not modify contact properties (selectivity) at the cathode. Under these circumstances the current density curve is mainly defined by the recombination processes taking place in the bulk of the active layer. In contrast, transport of carriers and contact selectivity are limiting factors for the PTB7:PC 60 BM-based solar cells. For this blend, best efficiencies are obtained with low active layer thickness, minimizing undesired resistance to transport, and high fullerene ratio, enhancing the amount of fullerene at the cathode leading to decreased leakage currents. Alternatively, the use of additives can also be used to manipulate the morphology and improve the contact selectivity. Thus, additives mainly result in the increment of the photogenerated and extracted charges.

\section{Experimental}

\section{Materials}

The following materials were used as received: P3HT (American Dye Source, Inc.), PTB7 (1-material), $\mathrm{PC}_{60} \mathrm{BM}$ (Nano-C), $\mathrm{PC}_{70} \mathrm{BM}$ (Nano-C), PEDOT:PSS (Al 4083 from H. C. Stark), chlorobenzene (Scharlau), ortho-dichlorobenzene (Sigma-Aldrich), and diiodooctane (Sigma-Aldrich). Blends of donor:acceptor were prepared prior to device fabrication. $\mathrm{P} 3 \mathrm{HT}: \mathrm{PC}_{60} \mathrm{BM}$ solutions (ratio $1: 1$ ) were prepared in chlorobenzene with an initial donor concentration of $15 \mathrm{mg} \mathrm{ml}{ }^{-1}$. Likewise, PTB7:PC 60 BM solutions were prepared in ortho-dichlorobenzene with an initial donor concentration of either $18 \mathrm{mg} \mathrm{ml}^{-1}$ or $24 \mathrm{mg} \mathrm{ml}^{-1}$ depending on the desired final film thickness. All solutions were heated overnight at $85{ }^{\circ} \mathrm{C}$. In order to be able to fairly compare the performance of both polymers, no diiodooctane (DIO) was used for the processing of PTB7. Furthermore, $\mathrm{PC}_{60} \mathrm{BM}$ was used instead of $\mathrm{PC}_{70} \mathrm{BM}$ as an electron acceptor. These two facts explain the poorer photovoltaic performance of PTB7 based devices in comparison to previously published studies with the same cell architecture. ${ }^{1}$ In contrast, we can be sure that the differences observed in the working mechanisms of solar cells are inherent to the intrinsic properties of the polymers and not related to different processing conditions. In addition, optimized devices containing PTB7 were obtained with the use of diiodooctane (DIO) and the replacement of $\mathrm{PC}_{60} \mathrm{BM}$ with the higher light absorbing acceptor $\mathrm{PC}_{70} \mathrm{BM}$. PTB7 (10 mg) and $\mathrm{PC}_{70} \mathrm{BM}(15 \mathrm{mg})$ were initially dissolved in $\mathrm{CB}$ inside a nitrogen glove-box $(970 \mu \mathrm{l})$. The solution was left stirring overnight at $60{ }^{\circ} \mathrm{C}$. After $24 \mathrm{~h}$ the corresponding amount of DIO $(30 \mu \mathrm{l})$ was added. The new solution was stirred $1 \mathrm{~h}$ at $70{ }^{\circ} \mathrm{C}$

Table 1 Device photovoltaic parameters measured under an illumination of $100 \mathrm{~mW} \mathrm{~cm}{ }^{-2}$ (AM1.5 G). *PC ${ }_{70} B M$ and DIO were used as acceptor molecule and additive, respectively

\begin{tabular}{lllllll}
\hline Device & Donor & D : A Ratio & Thickness $(\mathrm{nm})$ & $V_{\text {oc }}(\mathrm{V})$ & $J_{\text {sc }}\left(\mathrm{mA} \mathrm{cm}^{-2}\right)$ & FF \\
\hline 1 & P3HT & $1: 1$ & 100 & 0.57 & 7.56 & 0.69 \\
2 & P3HT & $1: 1$ & 270 & 0.58 & 8.03 & 2.95 \\
3 & PTB7 & $1: 1.5^{*}$ & 100 & 0.72 & 14.28 & 0.67 \\
4 & PTB7 & $1: 3$ & 100 & 0.72 & 10.31 & 0.65 \\
5 & PTB7 & $1: 1$ & 100 & 0.78 & 12.23 & 0.66 \\
6 & PTB7 & $1: 1$ & 340 & 0.78 & 4.28 & 0.47 \\
\end{tabular}


just before film deposition. Under these conditions the photovoltaic performance of PTB7-based devices is similar to previously published studies with the same cell architecture. ${ }^{1}$

\section{Device preparation}

Devices were fabricated in the configuration ITO/PEDOT:PSS/ Active layer/Ca/Ag. Structured indium tin oxide (ITO) coated glass substrates were cleaned in subsequent acetone and isopropyl alcohol ultrasonic baths followed by 5 minutes of UV ozone treatment. A thin layer of PEDOT:PSS was spin coated on the substrates to smooth the ITO surface and to act as the hole selective contact. Substrates were annealed at $120{ }^{\circ} \mathrm{C}$ for 10 minutes in air. Photoactive layers were cast either by spin coating at $1500 \mathrm{rpm}(100 \mathrm{~nm})$ or Dr Blade (thicker active layers). For Dr Blade deposition the plate temperature was set at $65{ }^{\circ} \mathrm{C}$ and the blade speed was set at $50 \mathrm{~mm} \mathrm{~s}^{-1}\left(\mathrm{P}_{3} \mathrm{HT}: \mathrm{PC}_{60} \mathrm{BM}\right.$, $270 \mathrm{~nm}$ ) or $30 \mathrm{~mm} \mathrm{~s}^{-1}$ (PTB7:PC $\left.{ }_{60} \mathrm{BM}, 340 \mathrm{~nm}\right)$. P3HT:PC ${ }_{60} \mathrm{BM}$ devices were annealed at $140{ }^{\circ} \mathrm{C}$ for 15 minutes in a nitrogen atmosphere. Finally, the cathode was thermally evaporated (10 nm of $\mathrm{Ca}$ and $100 \mathrm{~nm}$ of $\mathrm{Ag}$ ) at a base pressure of $4 \times$ $10^{-6}$ mbar to define an OPV active area of $9 \mathrm{~mm}^{2}$. Devices were encapsulated with an epoxy resin and a glass cover and were characterized outside the glovebox.

\section{Device characterization}

Current density-voltage $(J-V)$ characterization was performed with a Keithley 2420 Source-Measure Unit under $100 \mathrm{~mW} \mathrm{~cm}^{-2}$ AM1.5G illumination. The calibration of the light intensity was carried out with an NREL certified monocrystalline silicon photodiode. Film thicknesses were measured using a Dektak 150 surface profilometer. The charge extraction setup consist of a white light LED ring from $\operatorname{LUXEON}_{(\mathrm{R})}$ Lumileds; these are focussed onto the devices that are held in open circuit equilibrium. Devices are connected to a DC power supply and a function generator TGP110. The light is switched off and the circuit is temporally closed, while charges are forced to pass through an oscilloscope TDS 2022 from Tektronix@ that registers the drop in voltage between a resistance of $50 \Omega$. In TPV measurements devices are connected to the $1 \mathrm{M} \Omega$ input terminal of the same oscilloscope and the background illumination was obtained from a ring of 6 white LEDs from LUX$\operatorname{EON}_{(\mathrm{R})}$. The small perturbation $(2 \mathrm{mV})$ was applied through a light pulse $\left(\mathrm{N}_{2}\right.$ laser nominal wavelength, $50 \mathrm{~ns}$ pulses). The polaron-recombination rate was calculated for illumination intensities ranging from 0.1 sun to 1 sun. Impedance spectra were recorded using an Autolab PGSTAT-30 equipped with a frequency analyzer module. A small voltage perturbation $(20 \mathrm{mV}$ rms) was applied at frequencies from $1 \mathrm{MHz}$ to $1 \mathrm{~Hz}$. Measurements were carried out under 1 sun light intensity calibrated with a monocrystalline silicon photodiode sweeping the DC voltage in the range 0 to $V_{\mathrm{oc}}$. For measurements carried out at $V_{\mathrm{oc}}$ conditions light intensity was modified by using metallic perforated sheets so the light source spectrum is not modified. Recombination resistance $R_{\text {rec }}$ and chemical capacitance $C_{\mu}$ were directly extracted from the low-frequency region as previously reported..$^{16}$

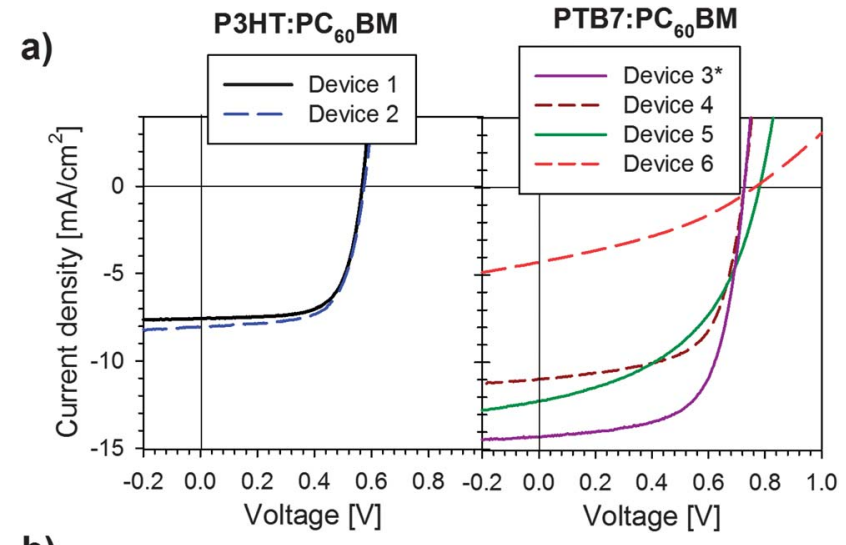

b)

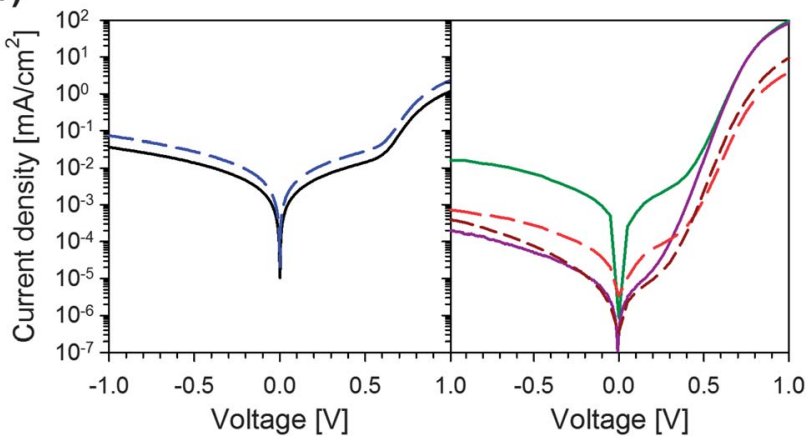

Fig. 1 Current density-voltage characteristics of devices fabricated with P3HT:PC 60 BM and PTB7:PC 60 BM measured: (a) under 1 sun light intensity and (b) diode curve under dark conditions. Increasing the active layer thickness for P3HT:PC ${ }_{60} B M$ does not considerably affect the device performance and dark curve. However, modification of the active layer thickness or donor/acceptor ratio in the PTB7:PC ${ }_{60} B M$ system dramatically alters the device characteristics. ${ }^{*} \mathrm{PC}_{70} \mathrm{BM}$ and $\mathrm{DIO}$ were used as the acceptor molecule and additive, respectively.

\section{Results and discussion}

\subsection{Current-voltage characteristics}

The current density-voltage $(J-V)$ curves of devices fabricated with the configuration ITO/PEDOT:PSS/Active layer/Ca/Ag are shown in Fig. 1 and the key parameters are listed in Table 1. It is clear that an increase in the active layer thickness in devices fabricated with $\mathrm{P} 3 \mathrm{HT}: \mathrm{PC}_{60} \mathrm{BM}$ does not show significant differences in either Fill Factor (FF) or open circuit potential $\left(V_{\mathrm{oc}}\right)$. Only slight variations on the generated photocurrent are observed similar to that reported by other authors. ${ }^{21}$ Then, a three-fold increase in the active layer thickness using doctor blade coating does not limit the device performance, implying that transport of carriers is efficient for the blend P3HT: $\mathrm{PC}_{60} \mathrm{BM}$. On the other hand, the $J-V$ curves of $\mathrm{PTB} 7: \mathrm{PC}_{60} \mathrm{BM}$ are in marked contrast to those observed for P3HT: $\mathrm{PC}_{60} \mathrm{BM}$. The best device performance is obtained with a $1: 1.5$ donor : acceptor ratio using DIO as an additive and $\mathrm{PC}_{70} \mathrm{BM}$ as an acceptor molecule, similar to previously reported results. ${ }^{1}$ In the absence of an additive and using $\mathrm{PC}_{60} \mathrm{BM}$ as an acceptor molecule (Device 4) the photocurrent is significantly reduced by $4 \mathrm{~mA} \mathrm{~cm}{ }^{-2}$. On the other hand, if the donor : acceptor ratio is adjusted to $1: 1$ (Device 5) to have a fair comparison with the $\mathrm{P} 3 \mathrm{HT}: \mathrm{PC}_{60} \mathrm{BM}$ system, the photocurrent is 
increased by about $2 \mathrm{~mA} \mathrm{~cm} \mathrm{~cm}^{-2}$ but the FF decreases sharply from 0.66 to 0.47 . As will be explained below the reduced FF for Device 5 is due to an enhanced leakage current. Additionally, if we compare thin and thick devices fabricated with $\mathrm{PTB} 7: \mathrm{PC}_{60} \mathrm{BM}$ with the same donor : acceptor ratio (Devices 5 and 6), a dramatic decrease in both photocurrent and FF is observed for the thick active layer. This dependency with active layer thickness strongly suggests that the system is limited by the transport of carriers through the bulk of the active layer. Interestingly, the $V_{\mathrm{oc}}$ remains almost invariant for the different device PTB7: $\mathrm{PC}_{60} \mathrm{BM}$ thicknesses.

Regardless of the deposition method and active layer thickness used both devices fabricated with P3HT:PC ${ }_{60} \mathrm{BM}$ show similar diode curve shapes under dark conditions (Fig. 1b). On the other hand, when we compare devices fabricated with PTB7: $\mathrm{PC}_{60} \mathrm{BM}$ diode curves differ not only in the shape but also in the photovoltaic parameters obtained. Then, Device 5 fabricated with a donor : acceptor ratio of $1: 1$ and low active layer thickness shows an enhanced leakage current at $-1 \mathrm{~V}$ of up to two orders of magnitude and a considerably higher current at forward bias. These results may explain as a first approximation the reduced FF observed for Device 5. However, it is important to note that due to the complexity of the blend and to the different morphological changes taking place during device processing the $J-V$ curve by itself does not provide conclusive evidence on why some devices perform better than others. The use of further characterization techniques is needed to support any conclusion and this will be the subject of our discussion in the following sections.

\subsection{Electrical losses in the bulk material}

3.2.1. Resistive processes. Impedance spectroscopy (IS) is an electrical technique that measures resistive and capacitive processes in operating devices. In a typical IS measurement under constant illumination we apply a dc voltage bias superimposed on a small ac voltage perturbation, and measure the differential current output. The frequency range of the ac voltage is wide enough $(1 \mathrm{~Hz}$ to $1 \mathrm{MHz})$ to obtain information on processes with a wide range of characteristic response times. These measurements are carried out at different dc voltages to cover the entire region where a working OPV is operating (a slightly wider range than $0 \mathrm{~V}$ to $V_{\mathrm{oc}}$ ). If all physical processes involved in device operation obey different characteristic times/ frequencies IS can provide simultaneous information on charge storage, ${ }^{22}$ carrier lifetimes, ${ }^{23}$ recombination kinetics and resistive processes limiting the device performance in an operating solar cell. ${ }^{13}$ The IS spectra of $\mathrm{P} 3 \mathrm{HT}: \mathrm{PC}_{60} \mathrm{BM}$ devices measured at $100 \mathrm{~mW} \mathrm{~cm} \mathrm{~cm}^{-2}$ light illumination displayed two arcs for both devices (Fig. 2a, left). The total resistance measured by IS is related to the derivative of the $J-V$ plot at a given dc voltage as

$$
R_{\mathrm{tot}}=\left(\frac{\partial J}{\partial V}\right)^{-1}
$$

The low frequency response here is dominant and has been associated previously with recombination processes (Fig. 2a, left). ${ }^{5}$ Indeed we noted that at high applied voltages the
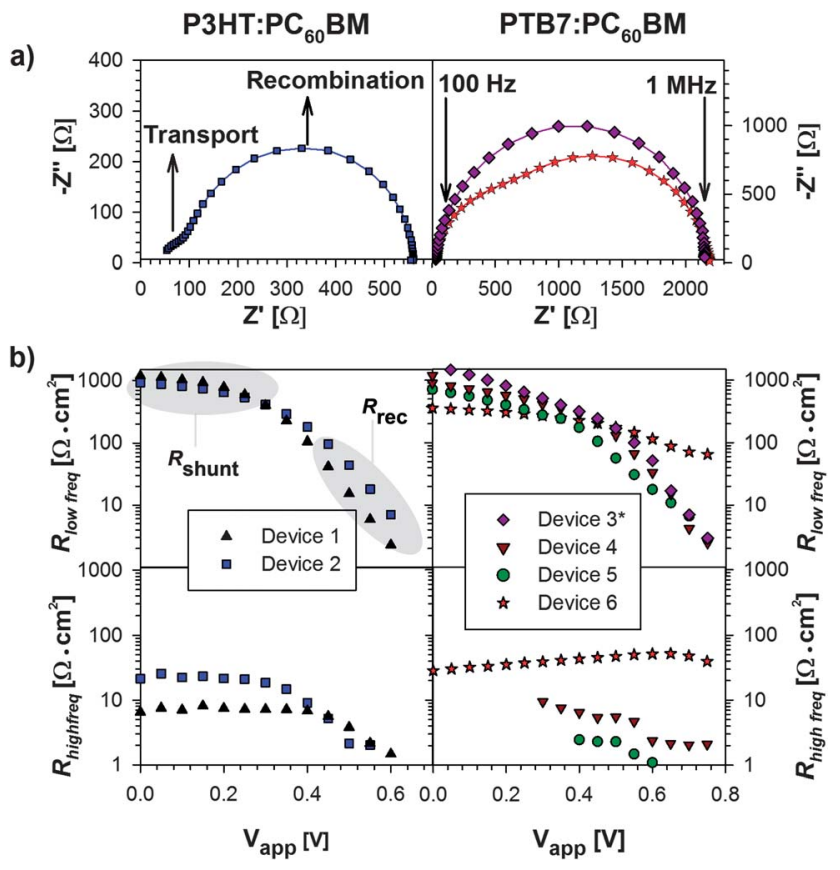

Fig. 2 (a) Two representative examples (Devices 2, 3, and 5) that illustrate the differences in the IS response for the two systems under study $\mathrm{P} 3 \mathrm{HT}: \mathrm{PC}_{60} \mathrm{BM}$ and PTB7:PC 60 BM measured at 1 sun light intensity and at applied voltages of 0.45 and $0.55 \mathrm{~V}$, respectively. (b) Fitted resistance data of all devices measured at 1 sun light intensity using the equivalent circuit discussed in the main text. Responses dominated by either shunt or recombination resistances have been highlighted for $\mathrm{P} 3 \mathrm{HT}: \mathrm{PC}_{60} \mathrm{BM}$. ${ }^{*} \mathrm{PC}_{70} \mathrm{BM}$ and $\mathrm{DIO}$ were used as acceptor molecule and additive, respectively.

response is connected to the recombination kinetics of the system (Fig. 2b, left). Alternatively, in the low applied bias region the IS response is likely dominated by the shunt resistance as a consequence of unavoidable leakage currents. ${ }^{22}$ The recombination resistance $\left(R_{\mathrm{rec}}\right)$ is related to the charge carrier recombination flux following the expression ${ }^{5,12,24}$

$$
R_{\mathrm{rec}}=\left(\frac{\partial J_{\mathrm{rec}}}{\partial V_{\mathrm{F}}}\right)^{-1}
$$

Here, $J_{\text {rec }}$ is a recombination current and $V_{\mathrm{F}}$ stands for the rise of the quasi-Fermi levels, and it is derived by subtracting the series resistance potential drop from the applied voltage,

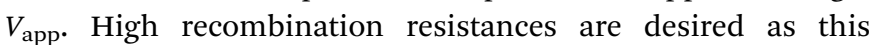
represents the opposition of the system to electrical losses caused by recombination. Alternatively, the high frequency response has previously been ascribed to issues related to transport of carriers in the bulk of the active layer and possesses a total current loss for the device performance. ${ }^{13}$ For the analyzed systems data can be fitted to a simple equivalent circuit as that defined by two resistance-capacitor subcircuits (RC) connected in series (see ESI $\dagger$ ). The high frequency response will be analysed here in detail and the low frequency response will be discussed in a subsequent section.

In ideal bulk heterojunction solar cells the polymer/fullerene blend shows adequate film morphology where acceptor molecules properly intercalate into the polymer phase leading to an improvement of the carrier transport. ${ }^{25,26}$ Here the 
P3HT:PC ${ }_{60} \mathrm{BM}$ system has been optimized by a fine control of the film drying process. Therefore, the resistance observed at high frequencies is most likely related to transport properties $\left(R_{\mathrm{tr}}\right)$. This is a parasitic loss for the device performance, and it is found to be about two orders of magnitude lower than those observed for the low frequency resistance ( $R_{\text {rec }}$, see Fig. $2 \mathrm{~b}$ ). Typical series resistances $\left(R_{\text {series }}\right)$ observed for "good" performing OPV devices are in the range of 1-20 $\Omega \mathrm{cm}^{2} .^{5,27}$ As the measured values at high frequency are within this range we can conclude that none of the P3HT: $\mathrm{PC}_{60} \mathrm{BM}$ devices studied are seriously limited by issues related to transport of carriers within the bulk of the active layer. Note that the high-frequency resistance at low voltages scales with the active layer thickness as expected. At high voltages, it is slightly lower for the thick active layer (Device 2) in comparison with the thin active layer (Device 1). However, it is important to highlight that these small differences $\left(\sim 2 \Omega \mathrm{cm}^{2}\right)$ may arise from small variations in the morphology of the blends. It should also be pointed out that for optimized devices it is also possible that the measured resistance at high frequency may be related to the external interfaces and not to the transport properties of the blend, in any case these are very small.

Similarly, impedance spectra of the optimized device fabricated with PTB7:PC ${ }_{70} \mathrm{BM}$ using DIO as an additive show mainly one arc (Fig. 2a, right) indicating that transport of carriers is not impeded. On the other hand, un-optimized devices (Devices 4-6) clearly show two arcs (Fig. 2a, right). In comparison with optimized devices, differences in morphology are expected to have an important impact in the exciton dissociation rates and charge transport properties. ${ }^{28}$ Both thin devices (Devices 4 and 5) show low high-frequency resistance, on the order of 2$10 \Omega \mathrm{cm}^{2}$, indicating that at this active layer thickness transport of carriers again is not an issue as in the case of P3HT: $\mathrm{PC}_{60} \mathrm{BM}$. However the noticeable difference in FF is a consequence of the high leakage current observed for Device 5. The transport resistance scales with the active layer thickness and Device 6 shows the highest resistances, on the order of $20-60 \Omega \mathrm{cm}^{2}$. For this last cell, these values are very similar to those obtained for the resistance extracted from the low frequency region, especially at high voltages. This is the first evidence supporting the fact that an inefficient charge transport process stemming from a non-ideal morphology can enormously enlarge the series resistance and consequently enhance charge recombination (Fig. 2).

3.2.2. Charge storage and carrier extraction. We analyze here how the system is able to store charges ${ }^{22}$ by observing the capacitive experimental parameters obtained from the IS. For systems not severely limited by transport of carriers, the device capacitance observed at low frequencies of the IS spectra is governed by the chemical capacitance due to excess carriers $C_{\mu}{ }^{29,30}$ which is related to the change in the occupancy by electrons of the fullerene LUMO, as follows

$$
C_{\mu}=q^{2} L \frac{\mathrm{d} n}{\mathrm{~d} E_{\mathrm{Fn}}}
$$

Here the capacitance is given per unit area, and $n$ corresponds to the electron concentration, $q$ stands for the

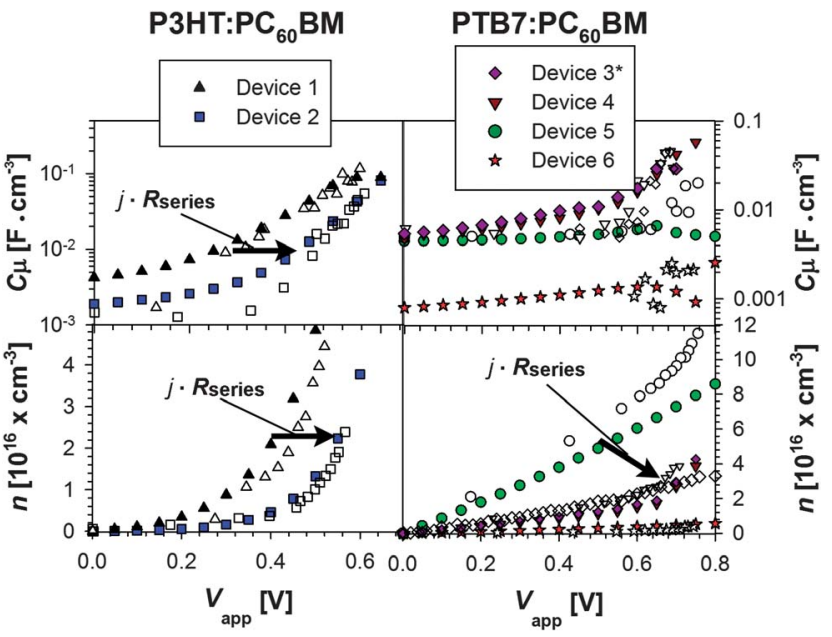

Fig. 3 Chemical capacitance and carrier density values as a function of the applied bias extracted from IS (filled symbols) and CE (open symbols). A voltage drop induced by the series resistance produces a shift in the chemical capacitance and carrier density curves. An exponential increase for both $\mathrm{P} 3 \mathrm{HT}: \mathrm{PC}_{60} \mathrm{BM}$ and thin PTB7:PC ${ }_{60} B M$ devices is observed. Device 6 does not show efficient charge extraction of carriers (voltage independent response) as a consequence of the transport limitation in the bulk of the active layer. ${ }^{*} \mathrm{PC}_{70} \mathrm{BM}$ and $\mathrm{DIO}$ were used as acceptor molecule and additive, respectively.

elementary charge, $L$ is the active layer thickness, and $E_{\mathrm{Fn}}$ is the electron Fermi level. An increase in $C_{\mu}$ is expected at forward bias as the density-of-states (DOS) occupancy progresses. ${ }^{22}$

Alternatively, we can use charge extraction (CE) measurements to infer the carrier density from charges extracted at the electrodes. For this time-domain technique cells are illuminated at open circuit conditions by a generated light pulse using different light intensities to produce different $V_{\text {oc }}$ (so-called light bias). Then, the cell is short-circuited and the decay in the photocurrent is measured and integrated to obtain the extracted charge. ${ }^{31,32}$ In other words, this time domain technique can be regarded as being complementary to IS. Thus, CE also provides information on how the system stores charges. ${ }^{33}$ As it is shown below both techniques IS and CE measure the carrier density yielding very similar results. This clearly reconfirms that they both measure the same physical phenomenon, CE in the time domain and IS in the frequency domain. Neither of the two techniques operates at open circuit conditions, then, the overall net current will not be null and the effect of series resistance will be taken into account using both techniques. Yet, small deviations are expected since the illumination conditions are clearly different for both measurements.

As expected for $\mathrm{P} 3 \mathrm{HT}: \mathrm{PC}_{60} \mathrm{BM}$ cells the observed behaviour with the applied bias of chemical capacitance and carrier density is similar for the two different active layer thickness devices (Fig. 3, left). At low applied bias the capacitive response is dominated by the dielectric properties of the active layer. As we increase the applied bias the DOS of the acceptor LUMO levels starts to be filled by electrons, and charge is stored with the consequent increase in $C_{\mu}$. A clear indication that we are measuring a chemical capacitance is the exponential increase over nearly two orders of magnitude, a rather distinct signature. 
Finally these stored charges can be collected at the electrodes in $\mathrm{CE}$. It is quite remarkable that in spite of the extremely similar $J-V$ characteristics for both $\mathrm{P} 3 \mathrm{HT}: \mathrm{PC}_{60} \mathrm{BM}$ (thin vs. thick devices) carrier density curves are shifted at any given applied potential, being the potential required to reach similar $C_{\mu}$ or carrier density values higher for the thick cell. It is then likely that the second resistance measured by IS at high frequency contributes as a source of series resistance and leads to a voltage drop. Therefore it needs to be included into the overall series resistance $\left(R_{\text {series }}\right)$. Then, we can calculate $R_{\text {series }}$ as

$$
R_{\text {series }}=R_{\mathrm{c}}+R_{\mathrm{tr}}
$$

where $R_{\mathrm{c}}$ is the series resistance due to contacts and wires calculated from the intercept of the high frequency arc with the $x$-axis and the zero value in the Nyquist plot. ${ }^{34}$ Indeed, we can correct the voltage for each data point to account for voltage losses due to the total series resistance of the device $\left(V_{\mathrm{F}}=V_{\text {app }}-j R_{\text {series }}\right)$ and we observe that both curves perfectly overlap (see ESI $\dagger$ ). ${ }^{5}$ This result clearly indicates that the voltage shift observed in $C_{\mu}$ and carrier density curves is indeed connected to the additional arc observed at high frequency $\left(R_{\mathrm{tr}}\right)$, which is an undesired resistance that contributes to the total series resistance of the device. Thus, as a first important result we must point out that $C-V$ measurements must take into account the additional electrical resistance present in thick devices such as those arising from mechanisms of transporting carriers towards the electrodes. In contrast, the information extracted may be misleading if the shift in the capacitance curves is directly attributed to either a shift in the tail or a broadening of the DOS.

A similar scenario is observed for devices based on the system PTB7:PC 60 BM. For the $100 \mathrm{~nm}$ OPVs (Devices 3, 4 and 5) both $C_{\mu}$ and carrier density increase with the applied voltage. Both CE and IS detect the storage of electrons, thanks to the low $\mathrm{R}_{\text {series }}$ observed in the range of $1-10 \Omega \mathrm{cm}^{2}$. On the other hand, for the device with the thick $\mathrm{PTB} 7: \mathrm{PC}_{60} \mathrm{BM}$ active layer (Device 6) the measured capacitance and carrier density are rather voltage independent. This result is strongly linked with the considerably large overall $R_{\text {series }}$ with values in the range of $20-60 \Omega \mathrm{cm}^{2}$. Additionally, since extraction and recombination are competing mechanisms the photocurrent is lowered as the transport resistance becomes larger.

It is interesting to note that above $0.65 \mathrm{~V}$ the chemical capacitance of Device 5 does not increase exponentially. Due to the implications on the recombination kinetics analysis in the next section we will now focus our attention to this feature. We have already stated that Device 5 shows increased leakage current in comparison to Devices 3 and 4 (Fig. 1b). Additionally by examining the impedance response as a function of the light intensity (Fig. 4) we observe that at low applied voltages, the resistive part arising from shunt resistance contributions decreases up to 4 orders of magnitude when the sample is illuminated compared to the measurement under dark conditions. These results are in clear contrast to those already published for the P3HT:PC ${ }_{60} \mathrm{BM}$ system in which the resistive part only varies by a factor of 3 when the device is illuminated from dark conditions to 1 sun. ${ }^{5}$

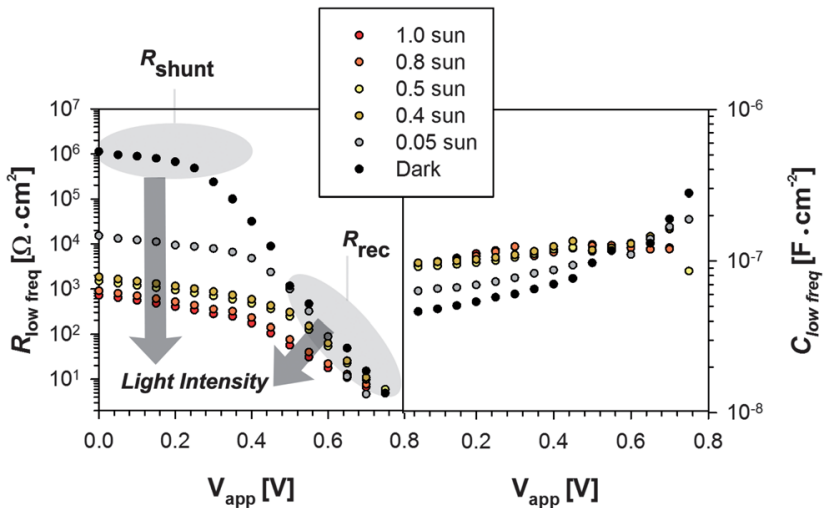

Fig. 4 Analysis of the low frequency response obtained using impedance spectroscopy for a device based on the PTB7:PC ${ }_{60}$ BM system (Device 5) measured under different light intensity conditions. The shunt resistance dramatically decreases with increasing light intensity and recombination kinetics is enhanced. Under high light intensity conditions leakage current is high, no carriers are stored carriers, and no chemical capacitance is observed.

For $\mathrm{PTB} 7: \mathrm{PC}_{60} \mathrm{BM}$ this large variation in the shunt resistance is a clear indication that the conductivity of the device is increasing with the increase of the light intensity resulting in an enhanced leakage current. This phenomenon is not well understood and could be originated by either polymer photodoping or light-induced reduction in contact selectivity. At a given voltage value the system is not able to store carriers and no chemical capacitance is observed. It is also interesting to note that in the high voltage region $\left(R_{\text {rec }}\right)$ the slope decreases with an increase in the light intensity. This result indicates that recombination kinetics is clearly enhanced under high light intensity conditions. In practical terms and in order to calculate the carrier density values in our following analysis we will extrapolate the values actually increasing exponentially. On the other hand, the voltage independent capacitance values observed for Device 6 raise the question as to whether the resistance extracted from the low frequency arc is a real recombination resistance for this device or just the response of a dielectric capacitor.

3.2.3. Recombination kinetics. Two techniques are most widely used to understand non-geminate recombination kinetics in working OPVs: transient photo-voltage (TPV) and IS. The TPV is a technique in which the device is held at open circuit conditions under continuous illumination. A weak, pulsed optical perturbation to this background illumination is then applied to the device, and the resultant perturbation to the voltage output of the device is monitored..$^{35,9}$ For each data point the device is illuminated at a different light bias and an overall zero current is extracted from the device whilst recording the resultant of the output perturbation. The fundamentals of IS have already been introduced using constant illumination. Alternatively, IS measurements using $V_{\text {oc }}$ conditions can also be carried out. It is very important to note that using $V_{\mathrm{oc}}$ conditions the net current flowing from the device is zero and the effect of the $R_{\text {series }}$ will then be null for both TPV and IS measurements. Then, resistive effects contributing to the total $R_{\text {series }}$ will be disregarded using $V_{\mathrm{oc}}$ conditions. 
Carrier lifetimes measured using TPV clearly show two different regions (see Fig. 5). At low charge density, corresponding to low light bias, the carrier lifetime is situated between $10^{-4}$ and $10^{-5}$ s. However, these results do not truly correspond to an effective carrier lifetime as the measured charge $(n)$ is close to the actual charge density due to the device geometrical capacitance $\left(\sim 50-150 \mathrm{nF} \mathrm{cm} \mathrm{cm}^{-2}\right)$. The measurements carried out at higher charge density $\left(n>10^{16} \mathrm{~cm}^{-3}\right)$ truly correspond to non-geminate recombination kinetics, and for our P3HT based solar cells are in the range of the microsecond timescale in agreement with those reported previously by our group and others. Of utmost importance is the fact that for P3HT devices IS shows perfect consistency not only in device open-circuit conditions but also when the measurement is carried out under $100 \mathrm{~mW} \mathrm{~cm}^{-2}$ light irradiation. On the other hand, devices made using PTB7 show substantial differences depending on their fullerene to polymer ratio and the use of additives. For an optimized active layer deposition process (Device 3) the situation is very similar to that observed for P3HT:PC ${ }_{60} \mathrm{BM}$ : TPV and IS results agree both at $V_{\text {oc }}$ and 1 sun light intensity conditions. Additionally, lifetimes are again on the order of microseconds. Whilst Device 4 (polymer : fullerene ratio $1: 3$ ) shows a straight line in log-log plot Device 5 (polymer : fullerene ratio $1: 1)$ shows two different regions. In this last case, the measurements displaying slow carrier lifetime cannot be attributed to non-geminate recombination kinetics but to the discharge of the geometrical capacitance. It is a matter of importance to highlight the fact that all measurements, either with TPV or IS, show identical slopes which indicate that the recombination reaction order will be identical independent of the technique used to measure it.

The response time representative of the recombination processes is calculated from the IS low frequency response as $\tau_{\text {rec }}=R_{\text {rec }} C_{\mu}$. Needless to say that in order to use this expression in a meaningful way the arc under analysis needs to be trulyoriginated by the charge recombination processes. The two main characteristics that need to be fulfilled are: recombination resistance values scale with device thickness, $R_{\text {rec }}$ and $C_{\mu}$ are volume dependent, and the chemical capacitance increases exponentially with the voltage. Device 5 shown previously in which the measured chemical capacitance does not increase exponentially cannot be regarded as a chemical capacitance as such and the characteristic time calculated from this arc will be originated from the discharge of a dielectric capacitor.

All results are in good agreement for P3HT: $\mathrm{PC}_{60} \mathrm{BM}$ measured by TPV and IS either at 1 sun light intensity (voltage sweep) or $V_{\text {oc }}$ conditions (different light intensities and no net current). Effective recombination times range in the order of $10^{-4}$ to $10^{-5} \mathrm{~s}^{-1}$. Selected devices are shown in Fig. 5 for clarity. All show decay dynamics that follow a power law trend as $\tau=$ $\tau_{\Delta \mathrm{n}_{0}} n^{-\alpha}$, suggesting that bimolecular recombination is the dominant charge loss mechanism. ${ }^{32,33}$

As shown in Fig. 5 all cells present a very similar decay and recombination dynamics. The order of the recombination mechanism ( $\alpha$, Table 2$)$ is slightly different for measurements carried out at 1 sun light intensity and $V_{\text {oc }}$ conditions in all cases between 1.4 and 2.8 being higher for $V_{\text {oc }}$ conditions. Similar

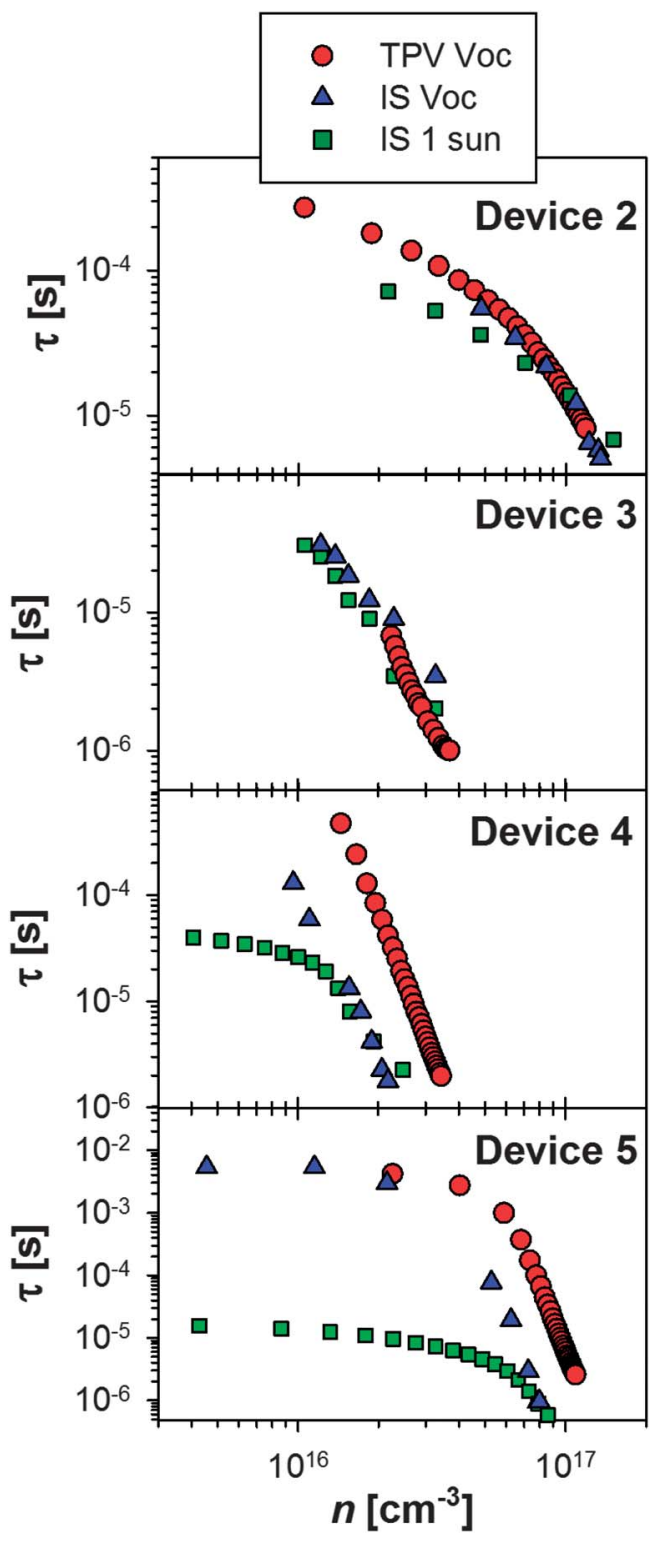

Fig. 5 Charge carrier lifetimes of representative devices based on either P3HT:PC ${ }_{60} B M$ or PTB7 at different carrier densities. TPV and IS measurements were performed under open circuit conditions and different illumination $\left(V_{o c}\right)$ or under constant illumination.

arguments apply for Device 3 fabricated with PTB7:PC ${ }_{70} \mathrm{BM}$. For Device 4 the variation in the effective recombination times varies significantly with the carrier density over three orders of magnitude under $V_{\text {oc }}$ conditions $\left(10^{-4}\right.$ to $\left.10^{-6} \mathrm{~s}^{-1}\right)$. Additionally, in the low carrier density region there are some evident discrepancies between measurements carried out under $V_{\text {oc }}$ conditions and those measured at 1 sun light intensity. Finally, Device 5 shows very different results depending on how the measurements have been carried out. Then, characteristic recombination times are on the order of $10^{-7} \mathrm{~s}^{-1}$ for IS measurements under 1 sun light intensity conditions and $10^{-3}$ $\mathrm{s}^{-1}$ for measurements at $V_{\mathrm{oc}}$ conditions.

Recombination order for all devices fabricated with the $\mathrm{PTB7}: \mathrm{PC}_{60} \mathrm{BM}$ (Devices 4-6) system calculated from 
measurements carried out under $V_{\mathrm{oc}}$ conditions provides values higher than 1 . In our case $\alpha=4.8-11$. The high value for the order of the recombination in $\mathrm{PTB} 7: \mathrm{PC}_{60} \mathrm{BM}$ devices can arise from different recombination pathways such as recombination of charges at the device contacts and carrier transport kinetics competing with carrier recombination kinetics. ${ }^{36}$ Since carrier transport is not compromised for thin devices the importance of the selective contacts results is key to minimize undesired losses at the device electrodes. For systems in which the contacts are not selective the leakage current is high and strongly depends on the light intensity. Then, measurements carried out at different light intensities $\left(V_{\mathrm{oc}}\right)$ suffer from the introduction of an additional parameter such as the differential variation of resistances and chemical capacitance with the light intensity. Overall, recombination times are artificially low at high light intensities and high at low light intensities, modifying the slope greatly. On the other hand, measurements at 1 sun light intensity are more reasonable $(\alpha=2.4-3.2)$. Due to the extremely low selectivity of the contact in Device 5 large variations in the leakage current are expected even at low variations in the applied voltage. Additionally, Device 6 lacks from an exponential increase in the chemical capacitance due to the large $R_{\text {series }}$ observed by direct consequence of the transport of carrier issues.

By comparison of results obtained by two different techniques and measured under different light intensity conditions we have provided the physical understanding of the conditions under which recombination kinetics affords physically meaningful results. In those situations in which the contacts are not selective the leakage current may be a dominant electrical loss mechanism and recombination processes taking place in the active layer will be masked.

\subsection{Origin of the high leakage current and contact selectivity}

It has previously been reported that the selectivity of the contact plays a very important role in the overall device efficiency. ${ }^{33,37-42}$ Provided we are using the same metal contact for devices 3-6, the resulting bulk morphology after film deposition and mainly the degree in which the active layer/cathode interface is covered by fullerene molecules will have a crucial impact in obtaining such a selectivity. Indeed, cathode interfaces poorly covered by fullerenes will provide low contact selectivity as inferred from the measured leakage current. Depending on the film deposition technique, conditions and the treatment afterwards, the donor : acceptor film profile across the active layer might vary substantially. In our set of experiments we either use spin coating or Doctor Blade coating to obtain either thin or thick active layers, respectively. Then, we can study how the active layer morphology/depositing process used to obtain different thickness devices will influence the final cathode coverage by fullerene and, hence, the contact selectivity.

The proportion of the cathode that is covered by fullerene can be measured in completed devices by using capacitancevoltage $(C-V)$ measurements in the dark as described previously by our group. ${ }^{42}$ Energy level equilibration at the active layer/

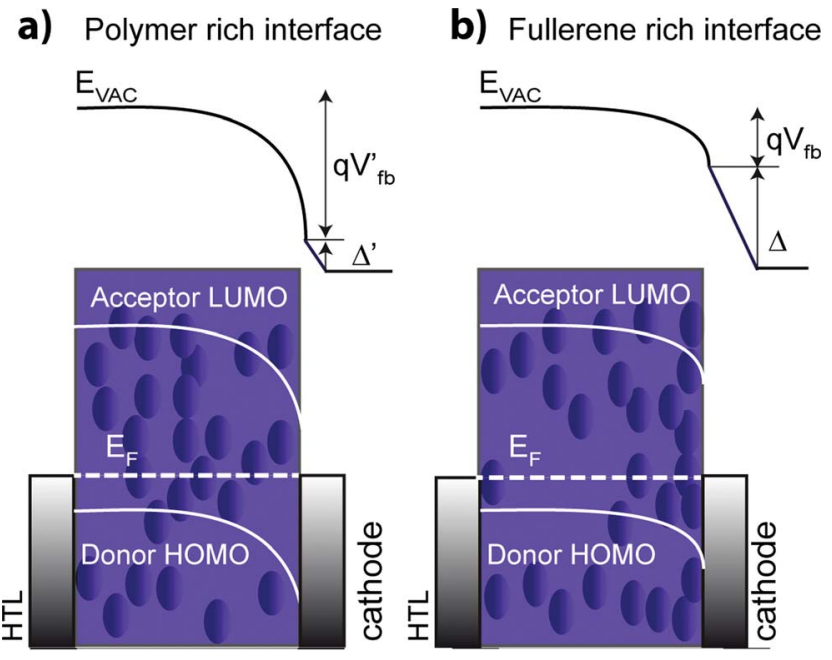

Fig. 6 Schematic representation of dipole layer formation and band bending contribution on the active layer/cathode interface equilibration. (a) Interfaces with high polymer coverage show large dipoles and low band bending contribution and (b) fullerenes covering the contact display small dipoles and large contribution from band bending.

cathode takes place by creation of a dipole layer and bulk band bending as shown in Fig. 6. The strength of the dipole and the band bending contribution will greatly depend on the material which is physically present at the interface, i.e., cathodes covered with a high proportion of fullerene show large dipoles and low band bending contribution. Indeed, we can carry out Mott-Schottky (MS) analysis to extract the applied potential that provides flat bands (flat band potential, $V_{\mathrm{fb}}$ ) and calculate the dipole strength. If two reference devices are used, one containing only pure polymer and a second that contains a blend with a high proportion of fullerene (donor : acceptor ratio $1: 6$ ), the $V_{\mathrm{fb}}$ for the extreme coverage case is accessible from MS analysis. Then, we can interpolate and calculate the degree of polymer/fullerene coverage for any blend between the two extreme cases. A complete analysis is carried out as ESI (Fig. SI $4 \dagger$ ) for the devices studied in this work and a summary is provided in Table 2 .

Then, we can identify that the lack of selectivity of the contact is a very important electrical loss mechanism during the

Table 2 Device characteristics as a result of IS, CE and TPV characterization ${ }^{2}$

\begin{tabular}{llllll}
\hline & $\alpha$ & $\alpha$ & $\alpha$ & & $\begin{array}{l}\text { Fullerene } \\
\text { coverage }[\%]\end{array}$ \\
\hline 1 & TPV $\left(V_{\text {oc }}\right)$ & IS $\left(V_{\text {oc }}\right)$ & IS $(1 \mathrm{sun})$ & $V_{\text {fb }}[\mathrm{V}]$ & \\
2 & 2.5 & 2.4 & 1.4 & 0.450 & 90 \\
3 & 2.8 & 2.7 & 1.5 & 0.377 & 95 \\
4 & 3.0 & 2.2 & 1.8 & 0.673 & 80 \\
5 & 6.7 & 5.1 & 2.5 & 0.633 & 85 \\
6 & 10.8 & 11.0 & 3.2 & 1.002 & 35 \\
& 5.1 & 4.8 & 2.4 & 0.770 & 65
\end{tabular}

${ }^{a}$ The estimated recombination slope $(\alpha)$ as measured using the different techniques described in this work. $V_{\mathrm{fb}}$ refers to the flat band potential or the applied potential at which band bending is totally overcome providing flat bands. 
fabrication process. It is clear that the donor : acceptor profile obtained using a different active layer thickness is highly material dependent. Different post-film deposition treatments as solvent annealing have allowed us to optimize the bulk morphology of the P3HT:PC ${ }_{60} \mathrm{BM}$ film. ${ }^{43}$ These devices show FF over $70 \%$ which means that morphology and contact selectivity are not an issue for the photovoltaic performance of the solar cells. There is therefore no significant hole injection from the cathode to the HOMO level of the donor, neither is there electron injection from the anode to the LUMO level of the polymer. In other words, there is no contact between the cathode and the $\mathrm{P} 3 \mathrm{HT}$, neither is there between the anode and the $\mathrm{PC}_{60} \mathrm{BM}$, thus showing good selectivity. This interpretation is supported by the experimentally measured fullerene coverage at the cathode shown in Table 2. Devices fabricated with P3HT:PC ${ }_{60} \mathrm{BM}$ only show a modest variation in the fullerene concentration at the cathode when increasing the device thickness and modifying the deposition process, in both cases the contact is highly selective and high FF values are obtained. Alternatively, the fullerene coverage in the PTB7:PC ${ }_{60} \mathrm{BM}$ system varies significantly with thickness. Although thick devices show better contact selectivity than the thin analogue the reduced ability to transport carriers through the thick active layer is mainly responsible for the reduction in device performance. This means that the same processing conditions that allow fine tuning of the resulting bulk morphology and give rise to good results for P3HT have to be modified for PTB7. As mentioned before, the use of additives such as DIO introduces beneficial changes in the morphology for the overall performance of this type of photovoltaic cell. The important implications from the lack of contact selectivity in the output measurements using different physical measurements have already been discussed in this work.

\section{Conclusions}

By studying two of the most general organic bulk heterojunction solar cells that provide very different morphological and physical properties we have been able to disentangle the different electrical loss pathways taking place in working devices. In particular we have been able to illustrate the effect of resistive losses present in devices that show issues related to transport of carriers in the bulk material and the effect induced by poorly selective contacts. When active layers are fabricated (thickness ca. $300 \mathrm{~nm}$ ) to fulfil effective light absorption requirements not only transport properties of the materials are important but also how the donor and acceptor materials are intermixed across the active layer thickness. We have shown that for the P3HT: $\mathrm{PC}_{60} \mathrm{BM}$ system the distribution of donor and acceptor is such that enables an efficient selectivity of the contacts. In addition, transport properties of thin and thick devices are very similar. In contrast, for a system such as $\mathrm{PTB} 7: \mathrm{PC}_{60} \mathrm{BM}$ higher proportions of fullerene (ratio 1:3) are required to reach selective contacts. Alternatively, additives can also be used in order to manipulate bulk morphology and improve contact selectivity. Although thick devices containing PTB7:PC ${ }_{60} \mathrm{BM}$ show better contact selectivity than the thin analogue the reduced ability to transport carriers through the active layer is mainly responsible for the reduction in device performance. The use of additives improves the charge generation and extraction and completely removes any issues related to transport of carriers. We conclude that morphology at the bulk of the active layer has a great influence both on photogeneration and charge transport. Similarly morphology and composition at the contact surface (fullerene surface coverage) affect the contact selectivity as the ability to extract one kind of charge and to block the other. This work also highlights that careful analysis needs to be carried out on the recombination kinetic results extracted from TPV and IS measured under $V_{\mathrm{oc}}$ conditions as unrealistic recombination orders can be obtained due to the influence of the leakage current and enhanced recombination at the device contacts leading to a poor device fill factor.

\section{Acknowledgements}

We thank the financial support from Generalitat Valenciana (Prometeo/2009/058, ACOMP/2009/056, ACOMP/2009/095, and ISIC/2012/008 Institute of Nanotechnologies for Clean Energies). EP thanks the ERC for the grant PolyDot, the ICIQ and ICREA for their financial support. The Spanish MINECO and the Catalan regional government are also acknowledged by the projects CTQ2012-18859 and 2009 SGR 207, respectively. RP, IE and JA thank the European Community's Seventh Framework Programme (FP7/2007-2013) under Grant no. 287818 of the $\mathrm{X} 10 \mathrm{D}$ project for providing financial support.

\section{References}

1 Y. Liang, Z. Xu, J. Xia, S.-T. Tsai, Y. Wu, G. Li, C. Ray and L. Yu, Adv. Mater., 2010, 22, E135-E138.

2 J. Peet, J. Y. Kim, N. E. Coates, W. L. Ma, D. Moses, A. J. Heeger and G. C. Bazan, Nat. Mater., 2007, 6, 497-500.

3 G. Yu, J. Gao, J. C. Hummelen, F. Wudl and A. J. Heeger, Science, 1995, 270, 1789-1791.

4 A. Maurano, R. Hamilton, C. G. Shuttle, A. M. Ballantyne, J. Nelson, B. O'Regan, W. Zhang, I. McCulloch, H. Azimi, M. Morana, C. J. Brabec and J. R. Durrant, Adv. Mater., 2010, 22, 4987-4992.

5 P. P. Boix, A. Guerrero, L. F. Marchesi, G. Garcia-Belmonte and J. Bisquert, Adv. Energy Mater., 2011, 1, 1073-1078.

6 F. C. Krebs, Sol. Energy Mater. Sol. Cells, 2009, 93, 394-412. 7 Z. He, C. Zhong, S. Su, M. Xu, H. Wu and Y. Cao, Nat. Photonics, 2012, 6, 591-595.

8 H.-L. Yip and A. K.-Y. Jen, Energy Environ. Sci., 2012, 5, 59946011.

9 A. Maurano, C. G. Shuttle, R. Hamilton, A. M. Ballantyne, J. Nelson, W. Zhang, M. Heeney and J. R. Durrant, J. Phys. Chem. C, 2011, 115(13), 5947-5957.

10 A. Sánchez-Díaz, L. Burtone, M. Riede and E. Palomares, J. Phys. Chem. C, 2012, 116, 16384-16390.

11 J. Albero, Y. Zhou, M. Eck, F. Rauscher, P. Niyamakom, I. Dumsch, S. Allard, U. Scherf, M. Kruger and E. Palomares, Chem. Sci., 2011, 2, 2396-2401. 
12 A. Guerrero, L. F. Marchesi, P. P. Boix, J. Bisquert and G. Garcia-Belmonte, J. Phys. Chem. Lett., 2012, 3, 1386-1392.

13 A. Guerrero, T. Ripolles-Sanchis, P. P. Boix and G. GarciaBelmonte, Org. Electron., 2012, 13, 2326-2332.

14 A. Sánchez-Díaz, M. Izquierdo, S. Filippone, N. Martin and E. Palomares, Adv. Funct. Mater., 2010, 20, 2695-2700.

15 F. Piersimoni, S. Chambon, K. Vandewal, R. Mens, T. Boonen, A. Gadisa, M. Izquierdo, S. Filippone, B. Ruttens, J. D'Haen, N. Martín, L. Liutsen, D. Vanderzande, P. Adriaensens and J. V. Manca, J. Phys. Chem. C, 2011, 115, 10873-10880.

16 P. P. Boix, M. M. Wienk, R. A. J. Janssen and G. GarciaBelmonte, J. Phys. Chem. C, 2011, 115, 15075-15080.

17 D. Credgington, R. Hamilton, P. Atienzar, J. Nelson and J. R. Durrant, Adv. Funct. Mater., 2011, 21, 2744-2753.

18 J. Bisquert and G. Garcia-Belmonte, J. Phys. Chem. Lett., 2011, 2, 1950-1964.

19 G. Li, Y. Yao, H. Yang, V. Shrotriya, G. Yang and Y. Yang, Adv. Funct. Mater., 2007, 17, 1636-1644.

20 W. Ma, C. Yang, X. Gong, K. Lee and A. J. Heeger, Adv. Funct. Mater., 2005, 15, 1617-1622.

21 G. Li, V. Shrotriya, Y. Yao and Y. Yang, J. Appl. Phys., 2005, 98, 043704.

22 G. Garcia-Belmonte, A. Guerrero and J. Bisquert, J. Phys. Chem. Lett., 2013, 4, 877-886.

23 G. Garcia-Belmonte, A. Munar, E. M. Barea, J. Bisquert, I. Ugarte and R. Pacios, Org. Electron., 2008, 9, 847-851.

24 A. Guerrero, L. F. Marchesi, P. P. Boix, J. Bisquert and G. Garcia-Belmonte, J. Phys. Chem. Lett., 2012, 3, 1386-1392.

25 S. J. Lou, J. M. Szarko, T. Xu, L. Yu, T. J. Marks and L. X. Chen, J. Am. Chem. Soc., 2011, 133, 20661-20663.

26 Y. Liang, Z. Xu, J. Xia, S. Tsai, Y. Wu, G. Li, C. Ray and L. Yu, Adv. Mater., 2010, 22, E135-E138.

27 R. Steim, The impact of interfaces on the performance of organic photovoltaic cells, KIT Scientific Publishing, 2010, p. 13.

28 M. R. Hammond, R. J. Kline, A. A. Herzing, L. J. Richter, D. S. Germack, H. Ro, C. L. Soles, D. A. Fischer, T. Xu,
L. Yu, M. F. Toney and D. M. DeLongchamp, ACS Nano, 2011, 5, 8248-8257.

29 J. Bisquert, D. Cahen, G. Hodes, S. Rühle and A. Zaban, J. Phys. Chem. B, 2004, 108, 8106-8118.

30 G. Garcia-Belmonte, P. P. Boix, J. Bisquert, M. Sessolo and H. J. Bolink, Sol. Energy Mater. Sol. Cells, 2010, 94, 366375.

31 C. G. Shuttle, A. Maurano, R. Hamilton, B. O'Regan, J. C. de Mello and J. R. Durrant, Appl. Phys. Lett., 2008, 93, 183501.

32 J. Ajuria, I. Etxebarria, E. Azaceta, R. Tena-Zaera, N. Fernandez-Montcada, E. Palomares and R. Pacios, Phys. Chem. Chem. Phys., 2011, 13, 20871-20876.

33 M. Bolognesi, A. Sanchez-Diaz, J. Ajuria, R. Pacios and E. Palomares, Phys. Chem. Chem. Phys., 2011, 13, 6105-6109. 34 F. Fabregat-Santiago, G. Garcia-Belmonte, I. Mora-Sero and J. Bisquert, Phys. Chem. Chem. Phys., 2011, 13, 9083-9118.

35 C. G. Shuttle, B. O'Regan, A. M. Ballantyne, J. Nelson, D. D. C. Bradley, A. J. Mello and J. R. Durrant, Appl. Phys. Lett., 2008, 92, 093311.

36 T. Kirchartz and J. Nelson, Phys. Rev. B: Condens. Matter Mater. Phys., 2012, 86, 165201.

37 R. Steim, F. R. Koglera and C. J. Brabec, J. Mater. Chem., 2010, 20, 2499-2512.

38 E. L. Ratcliff, B. Zacher and N. R. Armstrong, J. Phys. Chem. Lett., 2011, 2, 1337-1350.

39 R. Po, C. Carbonera, A. Bernardia and N. Camaioni, Energy Environ. Sci., 2011, 4, 285-310.

40 E. L. Ratcliff, B. Zacher and N. R. Armstrong, J. Phys. Chem. Lett., 2011, 2, 1337-1350.

41 A. Guerrero, L. F. Marchesi, P. P. Boix, S. Ruiz-Raga, T. Ripolles-Sanchis, G. Garcia-Belmonte and J. Bisquert, ACS Nano, 2012, 6, 3453-3460.

42 A. Guerrero, B. Dörling, T. Ripolles-Sanchis, M. Aghamohammadi, E. Barrena, M. Campoy-Quiles and G. Garcia-Belmonte, ACS Nano, 2013, 7, 4637-4646.

43 J. Ajuria, I. Etxebarria, W. Cambarau, U. Munecas, R. TenaZaera, J. C. Jimeno and R. Pacios, Energy Environ. Sci., 2011, 4, 453-458. 